\title{
Inhibiting, stabilising and probing the function of the Retromer endosomal trafficking complex through the novel macrocyclic peptides
}

\author{
Kai-En Chen ${ }^{1}$, Qian Guo ${ }^{1}$, Timothy A. Hill ${ }^{1}$, Yi Cui $^{2}$, Amy K. Kendall ${ }^{3}$, Natalya Leneva ${ }^{4}$, Zhe Yang $^{2}$, David P. Fairlie ${ }^{1}$, \\ Hiroaki Suga ${ }^{5}$, Lauren P. Jackson ${ }^{3}$, Rohan D. Teasdale ${ }^{2}$, Toby Passioura ${ }^{6}$, Brett M. Collins ${ }^{1}$ \\ ${ }^{1}$ The University of Queensland, Institute for Molecular Bioscience, St. Lucia, Queensland, 4072, Australia; \\ ${ }^{2}$ The University of Queensland, School of Biomedical Sciences, St Lucia, Queensland, 4072, Australia; \\ ${ }^{3}$ Department of Biological Sciences, Center for Structural Biology, Vanderbilt University, Nashville, TN 37232, USA; \\ ${ }^{4}$ Cambridge Institute for Medical Research, University of Cambridge, Cambridge, UK, \\ ${ }^{5}$ Department of Chemistry, Graduate School of Science, the University of Tokyo, 7-3-1 Hongo, Bunkyo-Ku, Tokyo 113-0033, Japan; \\ ${ }^{6}$ Sydney Analytical, the University of Sydney, Camperdown, New South Wales 2050, Australia.
}

\section{k.chen@imb.uq.edu.au}

The evolutionarily conserved Retromer complex (Vps35-Vps26-Vps29) is a master regulator responsible for endosomal membrane trafficking and signalling. It is known that mutations in Retromer can cause late-onset Parkinson's disease, and can also be hijacked by viral and bacterial pathogens during cellular infection. Seeking tools to modulate Retromer function would provide new avenues in understanding its function and the associated diseases. Here we employed the random nonstandard peptides integrated discovery (RaPID) approach to identify a group of macrocyclic peptides capable of binding to Retromer with high affinity. Our crystal structures show that five of the macrocyclic peptides bind to human Vps29 via a di-peptide Pro-Leu sequence. Interestingly, these peptides structurally mimic known interacting proteins including TBC1D5, VARP, and the bacterial effector RidL, and potently inhibit their interaction with Retromer in vitro and in cells. In addition, we found that these Vps29-binding macrocyclic peptides also mimic the binding between thermophilic yeast Vps29 and the unstructured N-terminal domain of Vps5. Disruption of this previously uncharacterized interaction by macrocyclic peptides negatively affect yeast Retromer, Vps5 and Vps17 to form stable heteropentameric complex. By contrast, mutagenesis and cryoEM show that macrocyclic peptide RT-L4 binds Retromer at the Vps35 and Vps26 interface, and it can act as a molecular chaperone to stabilise the complex with minimal disruptive effects on Retromer's ability to interact with its accessory proteins. Finally, using reversible cell permeabilization approach, we demonstrate that both the Retromer inhibiting and stabilizing macrocyclic peptides can specifically co-label Vps35-positive endosomal structures, and can be used as baits for purifying Retromer from cells and subsequent proteomic analyses. We believe these macrocyclic peptides can be used as a novel toolbox for the study of Retromer-mediated endosomal trafficking, and sheds light on developing novel therapeutic modifiers of Retromer function.

Keywords: Membrane trafficking; Retromer; macromolecular crystallography; CryoEM; cyclic peptide 